\title{
Optimization of the porcine adult skin-derived precursor cell isolation protocol
}

\author{
Haizea Iribar ${ }^{1}$, Begoña Castro-Feo², Iker Azcoitia-Ramsden², Naiara de Paz-Alonso², \\ Ander Izeta $^{1}$, Araika Gutiérrez-Rivera ${ }^{1^{*}}$ \\ ${ }^{1}$ Tissue Engineering Lab, Department of Bioengineering, Instituto Biodonostia, Hospital Universitario Donostia, San Sebastián, Spain; \\ *Corresponding Author: araika.gutierrez@,biodonostia.org \\ ${ }^{2}$ Histocell Tissue Engineering, Parque Tecnológico de Bizkaia, Derio, Spain
}

Received 13 November 2012; revised 14 December 2012; accepted 10 January 2013

\begin{abstract}
Neuronal and glial differentiation potential of skin-derived precursors is of great interest for clinical application in the treatment of neurodegenerative disease. In this sense, the pig model is a great candidate for the development of preclinical models. To the date, skin-derived precursor spheres have not been isolated from adult porcine skin. In order to optimize the protocol for isolating dermal precursor spheres from adult porcine skin, 15 porcine skin biopsies were subjected to three different processing protocols. Liberase-based digestion of ventral porcine skin gave rise to more cells with spherogenic capacity than other protocols and these spheres presented phenotypic and differentiation potential consistent with bona fide skin-derived precursor cells.
\end{abstract}

Keywords: Porcine; Dermal Precursors; SKPs; Clinical Application

\section{INTRODUCTION}

Skin-derived precursors (SKPs) are a population of neural crest-derived multipotent precursor cells present in both human and mouse dermis, distinct from mesenchymal and central nervous system stem cells [1-3]. They express genes characteristic of embryonic neural crest cells [1] and can be identified in vitro as non adherent cells isolated from the dermis that proliferate and selfrenew in response to growth factors FGF-2 and EGF. In vitro, SKPs may be differentiated into mesodermal lineages such as SMA+ smooth muscle cells and adipocytes, as well as into neural crest-derived progeny such as neurons and Schwann cells [1,2]. In vivo, SKPs derive from Sox $2+$ follicle-associated dermal precursors and show characteristics of dermal stem cells [4].

Neuronal and glial differentiation potential of SKPs is of great interest for clinical application in the treatment of neurodegenerative disease. However, development of safe clinical protocols usually requires preclinical validation of the in vitro results both in lower mammals (i.e rodents) and in a large animal model of disease. In this sense, the pig skin shares many physiological and anatomopathological similarities to its human counterpart. Dermal-epidermal thickness ratio ranges are very similar and both swine and human skin present well-developed rete ridges, dermal papillary bodies and abundant subcutaneous adipose tissue. Pigs and humans have relatively sparse body hair which progresses through the hair cycle independently of neighbouring follicles and, in contraposition to rodents, the mechanism of closure of partialthickness wounds proceeds largely through reepithelialization and not by wound contraction as in smaller mammals [5]. For these reasons, an improvement of SKP isolation and characterization from the pig model would be of great interest to push these cells forward to the clinic.

In 2004, Dyce et al. presented the first report of skinoriginated stem cells isolated from non-rodent animals [6]. They isolated SKPs from fetal porcine skin, by adapting the rodent method to larger animals [2]. Since then, a second group has replicated these findings [7], as reviewed in ref. [8]. More recently, Lermen et al. used adult porcine skin for the derivation of skin derived stem cell-like cells [9]. Similarly to SKPs, those cells stained positive for nestin and differentiated into mesodermal and neuronal lineages. However, they grew in attachment in serum-containing media and expressed mesenchymal stem cell related proteins, leaving the relationship to SKPs unclear. In summary, skin-derived precursor spheres have not been isolated as such from adult porcine skin [6-9].

\section{MATHERIALS AND METHODS}

\subsection{Cell Isolation and Culture}

A total of 15 skin biopsies (Table 1) of $5 \times 2 \mathrm{~cm}$ were obtained following the relevant legal and ethical guidelines, from anaesthetized female pigs of $45-50 \mathrm{~kg}$ body 
Table 1. Cell isolation efficiency in porcine skin biopsies.

\begin{tabular}{|c|c|c|c|c|c|c|c|}
\hline Biopsy Code & Protocol (P) & Donor Area & Enzyme & $\begin{array}{c}\text { Biopsy } \\
\text { Weight (g) }\end{array}$ & $\begin{array}{c}\text { Cell } \\
\text { Number } \\
\left({ }^{*} 10 \mathrm{E} 6\right)\end{array}$ & $\begin{array}{c}\text { Isolation } \\
\text { Efficiency } \\
\left.\text { (Cells/g }{ }^{*} 10 \mathrm{E} 6\right)\end{array}$ & Sphere Formation \\
\hline BC-3 & P1 & lower leg & $\mathrm{T} / \mathrm{E}$ & 4.34 & 3 & 0.69 & - \\
\hline BC-4 & P1 & lower leg & $\mathrm{T} / \mathrm{E}$ & 2.95 & 1.5 & 0.51 & - \\
\hline BC-5 & P1 & lower leg & $\mathrm{T} / \mathrm{E}$ & 3.13 & 2.2 & 0.70 & - \\
\hline BC001/11 & $\mathrm{P} 2$ & dorsal & $\mathrm{T} / \mathrm{E}$ & 3.23 & 1.1 & 0.34 & + \\
\hline $\mathrm{BC} 002 / 11$ & P2 & dorsal & $\mathrm{T} / \mathrm{E}$ & 2.88 & 1 & 0.35 & + \\
\hline $\mathrm{BC} 003 / 11$ & P2 & dorsal & $\mathrm{T} / \mathrm{E}$ & 3.15 & 0.4 & 0.13 & + \\
\hline BC004/11 & P2 & dorsal & $\mathrm{T} / \mathrm{E}$ & 2.95 & 0.8 & 0.27 & + \\
\hline $\mathrm{BC} 005 / 11$ & $\mathrm{P} 2$ & dorsal & $\mathrm{T} / \mathrm{E}$ & 2.95 & 0.4 & 0.14 & - \\
\hline $\mathrm{BC} 006 / 11$ & $\mathrm{P} 2$ & dorsal & $\mathrm{T} / \mathrm{E}$ & 1.61 & 0.7 & 0.43 & - \\
\hline BC007/11 & $\mathrm{P} 2$ & dorsal & $\mathrm{T} / \mathrm{E}$ & 1.85 & 0.8 & 0.43 & + \\
\hline BC0015/11 & P3 & ventral & LIBERASE & 1.97 & 3 & 1.52 & + \\
\hline BC0018/11 & P3 & ventral & LIBERASE & 0.84 & 0.4 & 0.48 & + \\
\hline ВС0019/11 & P3 & ventral & LIBERASE & 0.82 & 0.3 & 0.37 & + \\
\hline BC0020/11 & P3 & ventral & LIBERASE & 1.06 & 0.7 & 0.66 & + \\
\hline ВC0021/11 & P3 & ventral & LIBERASE & 0.77 & 1.1 & 1.43 & + \\
\hline
\end{tabular}

weight. Biopsies were stored in PBS or RPMI (Sigma) supplemented with $2 \%$ penicillin/streptomycin $(\mathrm{P} / \mathrm{S})$ and processed at a maximum 24 hours post-sacrifice, as previously described [10], with variations in the donor area and the disaggregating enzyme of choice. Biopsies were obtained from three different regions: lower leg, abdomen and back skin. To obtain cell suspensions, the tissue was cut into $1-2 \mathrm{~mm}$ pieces and incubated with trypsin/ EDTA (T/E, Sigma) or liberase DH (Roche). For protocol 1, lower leg skin was incubated with trypsin/EDTA overnight $(\mathrm{O} / \mathrm{N})$; for protocol 2, dorsal skin was incubated with T/E between 4 and 16 hours and for protocol 3 ventral skin was incubated with liberase between 24 48 hours. After the first digestion step, epidermis was removed from dermis and discarded. Dermis was then minced and incubated with the same enzyme as in the first step, at $37^{\circ} \mathrm{C}$. The sample was then centrifuged and the supernatant discarded. The remaining skin pieces were then mechanically dissociated and the cells obtained cultured in DMEM/F12 (Sigma) supplemented with methylcellulose ( $0.8 \%, \mathrm{R} \& \mathrm{D}), \mathrm{B} 27$ ( $2 \%$, Gibco), FGF (40 $\mathrm{ng} / \mathrm{ml}$, Gibco), EGF (20 ng/ml, Austral Biologicals), P/S and glutamine (Sigma) at a cell density of 90,000 cells/ $\mathrm{cm}^{2}$.

\subsection{Cell Differentiation}

For cell differentiation, 2000 cells were plated per well onto coverslips covered with extracellular matrix secreted by the $804 \mathrm{G}$ cell line [11], on 24-well culture dishes. Cells were let to proliferate in basal medium (BM) consisting of DMEM/F12 supplemented with B27, P/S, glutamine and 1\% FBS (Sigma) for 3-4 days. Then, cells were let to differentiate for 2 weeks in neural differentiation medium consisting of DMEM/F12 supplemented with $\mathrm{P} / \mathrm{S}$, glutamine, $\mathrm{N} 2$ ( $1 \%$, Invitrogen), forskolin (5 $\mathrm{uM}$, Sigma) and Heregulin $\beta-1$ (40 ng/ml, Peprotech).

\subsection{Immunofluorescence}

Spheres were seeded in 12-mm diameter-coverslips previously treated to improve adherence as above. Two hours later, dermospheres were fixed with $4 \%$ paraformaldehyde for 15 minutes and permeabilised in $0.2 \%$ Triton X-100 and 6\% FBS for 40 minutes. Differentiated cell cultures were fixed with $4 \%$ paraformaldehyde in PBS for 10 minutes and permeabilised in $0.5 \%$ Triton X100 for 10 minutes. Anti-nestin (Santa Cruz, polyclonal), vimentin (Sigma, clone v9), fibronectin (Sigma, polyclonal), SMA (Sigma, clone 1A4) and $\beta \mathrm{III}$ tubulin (Ab- 
cam, polyclonal) primary antibodies were used. Goat anti rabbit Alexa $488(\mathrm{H}+\mathrm{L})$ and Goat anti mouse Alexa 488 $(\mathrm{H}+\mathrm{L})$ (Molecular Probes) were used as secondary antibodies. Hoechst 33258 (Sigma, $1 \mu \mathrm{g} / \mathrm{ml}$ ) was used as nuclear counterstain and the slides were mounted on Vectashield (Vector laboratories).

\subsection{Quantification of Cell Differentiation}

To estimate percent cells differentiated to a given phenotype, 10 fields were counted per coverslip. Total cells were estimated through nuclear counts and cells in a given phenotype through counting of cells positive to the specific markers. Average of the 10 fields was calculated for each coverslip.

\subsection{Statistical Analyses}

One-way ANOVA tests, with Scheffé corrections, were used to calculate $\mathrm{p}$ values between different protocols. IBM SPSS Statistics Version 20 was used.

\section{RESULTS}

\subsection{Optimization of Dermal Precursor Sphere Isolation}

In order to assess the best protocol for isolating dermal precursor spheres from adult porcine skin, we subjected 15 porcine skin biopsies (Table 1) to three different processing protocols. All protocols isolated SKPs as previously described [10], with variations in the donor area

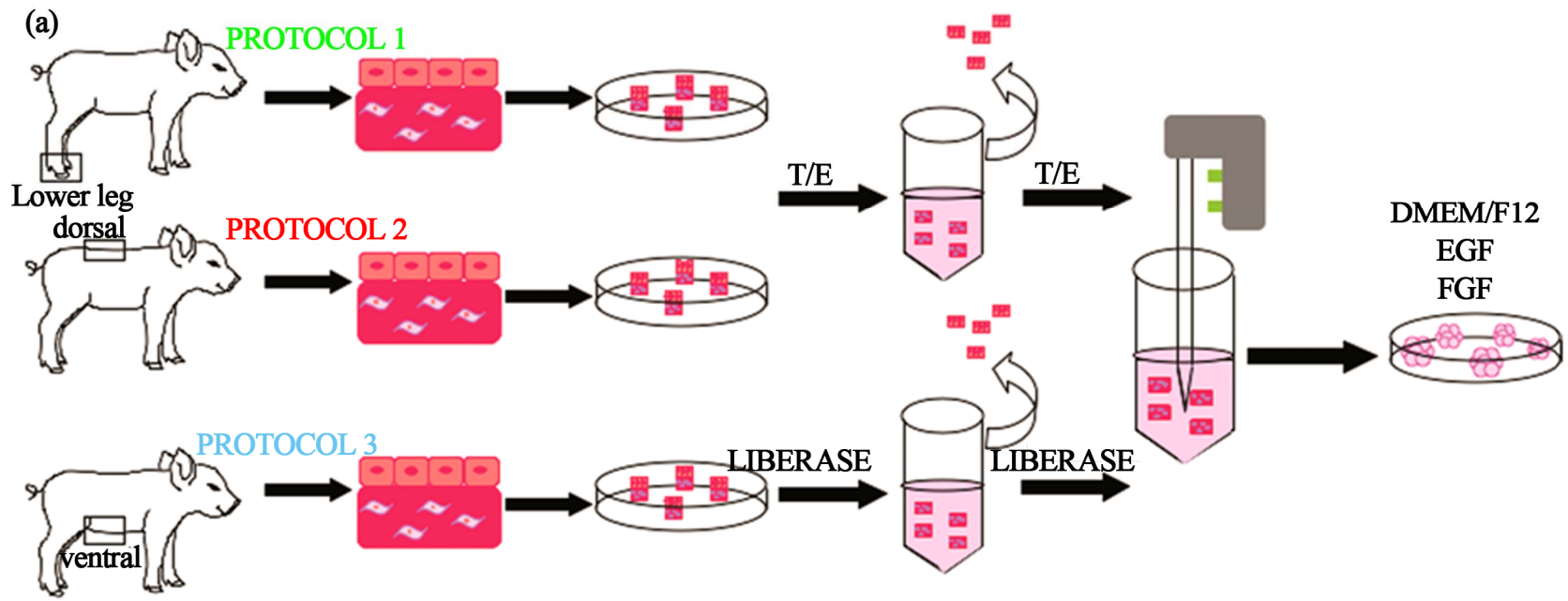

(b)

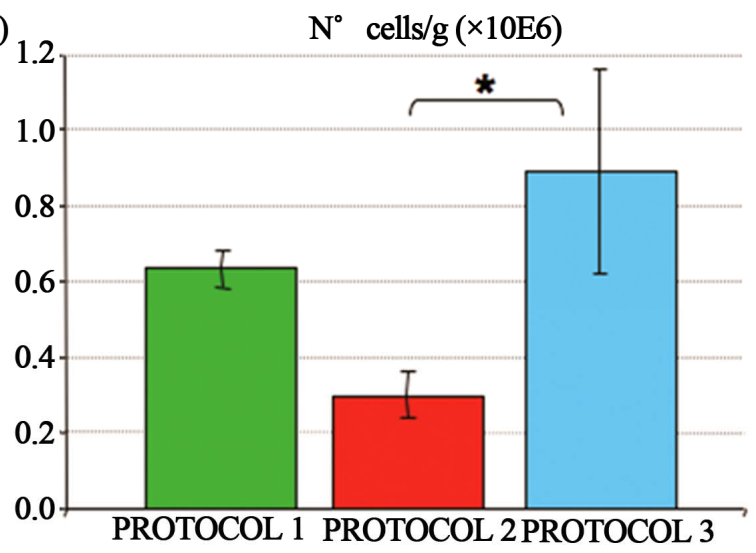

(c)

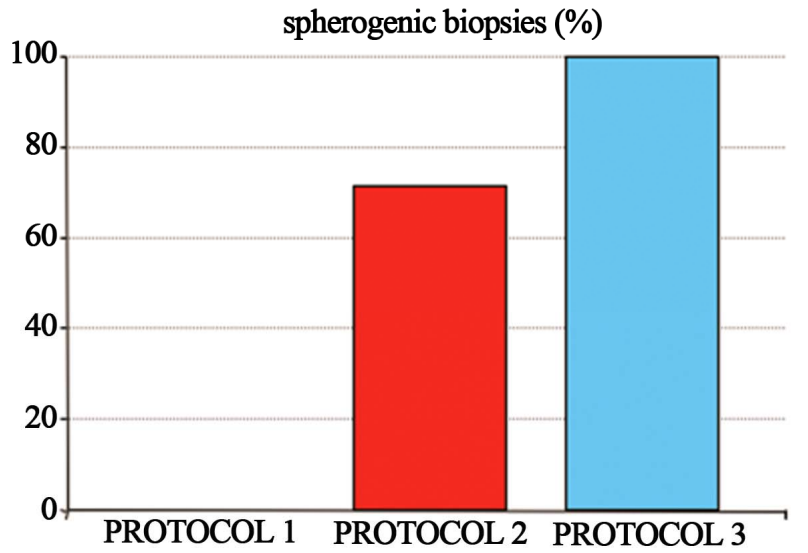

Figure 1. Optimization of skin-derived precursor isolation from porcine adult skin. (a) Schematic representation of skin processing protocols. In protocol 1, lower leg skin was incubated with trypsin/EDTA (T/E) overnight $(\mathrm{O} / \mathrm{N})$; in protocol 2, dorsal skin was incubated with $\mathrm{T} / \mathrm{E}$ between 4 and 16 hours and for protocol 3 ventral skin was incubated with liberase between 24 - 48 hours. After the first digestion step, the epidermis was separated from underlying tissue and discarded. The dermis was then minced and incubated with the same enzyme as in the first step, at $37^{\circ} \mathrm{C}$. The sample was then centrifuged and the supernatant discarded. The remaining skin pieces were mechanically dissociated and the cells suspension was cultured in sphere-forming medium; (b) Number of cells obtained per gram of tissue. Statistically significant differences $(p<0.05)$ were observed between protocol 2 and 3; (c) Percentage of biopsies giving rise to spheres under each protocol. 
and the disaggregating enzyme of choice (Figure 1). In protocol 1 and 2, Trypsin/EDTA (T/E) was used for skin disaggregation, while Liberase $\mathrm{DH}$ was used in protocol 3 . With respect to donor area, lower leg (protocol $1, \mathrm{n}=$ 3 ), dorsal (protocol 2, $\mathrm{n}=7$ ) and ventral skin (protocol 3, $\mathrm{n}=5$ ) were tested. The cell suspensions obtained were seeded at a fixed cell density of 90,000 cells $/ \mathrm{cm}^{2}$ in untreated culture plates, and grown in serum free proliferation medium consisting of DMEM/F12 supplemented with methylcellulose, B27, FGF, EGF, P/S and L-Glutamine (see Matherials and Methods). We first compared the number of cells per gram of tissue obtained in each protocol.

On average, protocol 3 yielded increased cellularity (Figure 1(b)) of the initial cell suspension, the difference to protocol 2 being statistically significant $(\mathrm{p}<0.05)$.

However, it was more important to see if the obtained cells give rise to spheres, which are enriched in precursor cells. Protocol 1 yielded no sphere (0/3 biopsies). Protocol 2 generated spheres in $71.43 \%$ of the cases (5/7 biopsies). Cells obtained under protocol 3 gave rise to spheres in $100 \%$ of the cases ( $5 / 5$ biopsies).

These results suggest that processing adult porcine ventral skin with Liberase DH generates SKP cultures in a reproducible manner, while ensuring maximum cellular yields are obtained per gram of tissue.

\subsection{Characterization}

To characterize the porcine adult SKPs (pSKPs) obtained by using the protocol of choice (protocol 3), porcine dermospheres (Figure 2(a)) were analyzed by immunofluorescence to detect SKP markers nestin, fibronectin and vimentin [1-3]. As expected, porcine dermospheres expressed all three canonical markers (Figures 2(b)-(d)).

To verify their multipotent differentiation capacity, pSKPs were put into differentiation in basal medium (consisting of DMEM/F12 supplemented with B27, P/S, glutamine and $1 \%$ FBS) and in neural differentiation medium (consisting of DMEM/F12 supplemented with P/S, glutamine, $\mathrm{N} 2$, forskolin, and Heregulin $\beta$ ) for 14 days (see Matherials and Methods). Differentiated pSKPs expressed both mesodermal (SMA, 16.4\%) and neural differentiation markers ( $\beta$ III tubulin, 25.2\%; Figure 2(e) and data not shown).

\section{CONCLUSION}

We have optimized the isolation of spherogenic cells from adult porcine skin. We conclude that liberase-based digestion of ventral porcine skin yields more cells with spherogenic capacity than other protocols and that these spheres presented phenotypic and differentiation potential consistent with bona fide skin-derived precursor cells.

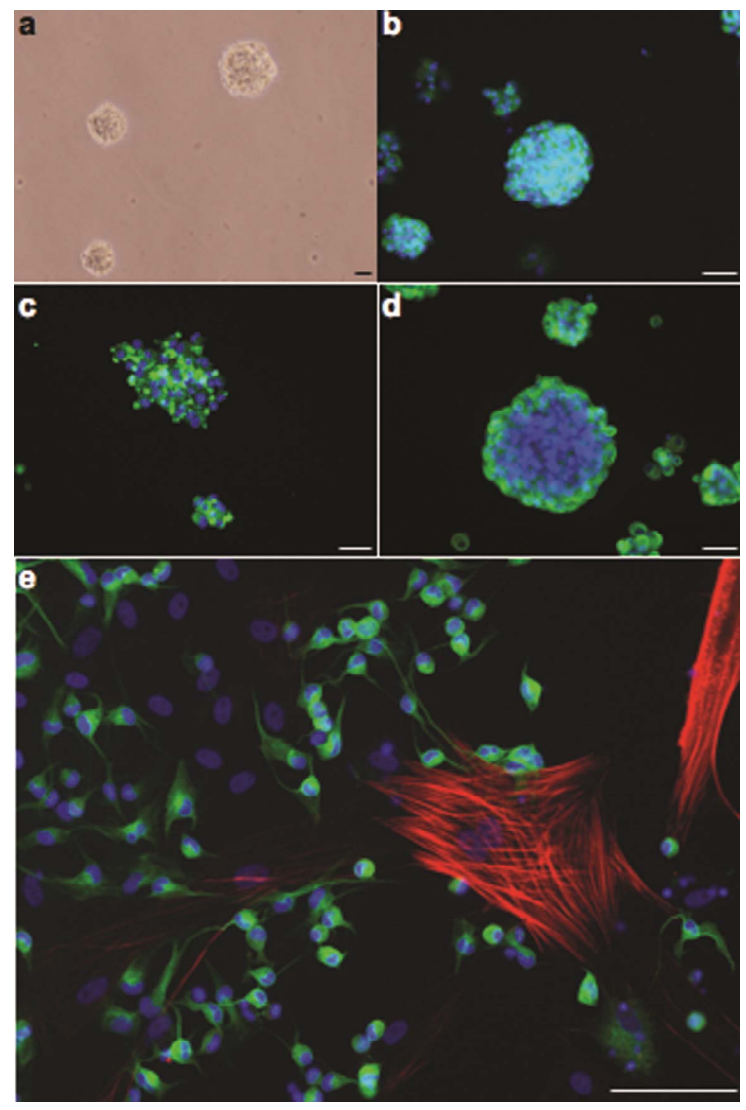

Figure 2. Characterization of adult porcine dermospheres. (a) Skin-derived precursor (SKP) cells derived from adult por- cine ventral skin grow as floating spheres in serum-free pro- liferation medium. Scale bar, $50 \mu \mathrm{m}$; (b-d) Characterization of primary dermospheres. Primary dermospheres were at- tached to coverslips and analyzed by immunofluorescence with anti-nestin (b), anti-fibronectin (c) and anti-vimentin (d) antibodies; (e) Char- acterization of cells grown for 2 weeks in neural differentiation medium. Positive immunoreactivity consistent with mesodermmal (smooth muscle actin, SMA+, red) and neural ( $\beta$ III tubu- lin+, green) differentiation was observed. Cell nuclei were counterstained with Hoechst (blue). Scale bars, $20 \mu \mathrm{m}$.

\section{ACKNOWLEDGEMENTS}

This work was financed by grants provided by the Department of Industry, Innovation, Commerce and Tourism of the Basque Government (Gaitek 10/002), Diputación Foral de Gipuzkoa (OF 53/2011) and Ministerio de Ciencia e Innovación (PI10/02871 and INNPACTO programs, IPT-300000-2010-17). A.I. was supported by the "Programa I3SNS" (CES09/015) from Instituto de Salud Carlos III and by Osakidetza (Spain).

\section{REFERENCES}

[1] Fernandes, K.J.L., McKenzie, I.A., Mill, P., Smith, K.M., 
Akhavan, M., Barnabé-Heider, F., Biernaskie, J., Junek, A., Kobayashi, N.R., Toma, J.G., Kaplan, D.R., Labosky, P.A., Rafuse, V., Hui, C.C. and Miller, F.D. (2004) A dermal niche for multipotent adult skin-derived precursor cells. Nature Cell Biology, 6, 1082-1093.

doi:10.1038/ncb1181

[2] Toma, J.G., Akhavan, M., Fernandes, K.J., Barnabé-Heider, F., Sadikot, A., Kaplan, D.R. and Miller, F.D. (2001) Isolation of multipotent adult stem cells from the dermis of mammalian skin. Nature Cell Biology, 3, 778-784. doi:10.1038/ncb0901-778

[3] Gago, N., Pérez-López, V., Sanz-Jaka, J.P., Cormenzana, P., Eizaguirre, I., Bernad, A. and Izeta, A. (2009) Agedependent depletion of human skin-derived progenitor cells. Stem Cells, 27, 1164-1172. doi:10.1002/stem.27

[4] Biernaskie, J., Paris, M., Morozova, O., Fagan, B.M., Marra, M., Pevny, L. and Miller, F.D. (2009) SKPs derive from hair follicle precursors and exhibit properties of adult dermal stem cells. Cell Stem Cell, 5, 610-623. doi:10.1016/j.stem.2009.10.019

[5] Sullivan, T.P., Eaglstein, W.H., Davis, S.C. and Mertz, P. (2001) The pig as a model for human wound healing. Wound Repair Regen, 9, 66-76. doi:10.1046/j.1524-475x.2001.00066.x

[6] Dyce, P.W., Zhu, H., Craig, J., Li, J. (2004) Stem cells with multilineage potential derived from porcine skin. Biochemical and Biophysical Research Communications, 316, 651-658.

[7] Zhao, M., Isom, S.C., Lin, H., Hao, Y., Zhang, Y., Zhao, J., Whyte, J.J., Dobbs, K.B. and Prather, R.S. (2009) Tracing the stemness of porcine skin-derived progenitors (pSKP) back to specific marker gene expression. Cloning Stem Cells, 11, 111-122. doi:10.1089/clo.2008.0071

[8] Zhao, M.T. and Prather, R.S. (2001) The multi-potentiality of skin-derived stem cells in pigs. Theriogenology, 75, 1372-1380. doi:10.1016/j.theriogenology.2010.06.010

[9] Lermen, D., Gorjup, E., Dyce, P.W., von Briesen, H. and Müller, P. (2010) Neuro-muscular differentiation of adult porcine skin derived stem cell-like cells. PLoS One, 5, e8968. doi:10.1371/journal.pone.0008968

[10] Biernaskie, J.A., McKenzie, I.A., Toma, J.G. and Miller, F.D. (2006) Isolation of skin-derived precursors (SKPs) and differentiation and enrichment of their Schwann cell progeny. Nature Protocols, 1, 2803-2812. doi:10.1038/nprot.2006.422

[11] Langhofer, M., Hopkinson, S.B. and Jones, J.C. (1993) The matrix secreted by $804 \mathrm{G}$ cells contains laminin-related components that participate in hemidesmosome assembly in vitro. Journal of Cell Science, 105, 753-764. 